This is a author version of

Raach, S. at al.: "Wake redirecting using feedback control

to improve the power output of wind farms,"

accepted at the American Control Conference ACC, USA, 2016.

\title{
Wake redirecting using feedback control to improve the power output of wind farms
}

\author{
Steffen Raach, David Schlipf, Friedemann Borisade and Po Wen Cheng
}

\begin{abstract}
In future, a wind turbine will not only be seen as a single systems operating independently, but also as a component of a larger interacting system, the wind farm. To increase the efficiency of a wind farm, two main concepts have been proposed: axial induction control and wake redirecting. This contribution focuses on the latter. Remote sensing technologies in wind energy applications have opened new ways to control wind turbines. In this contribution, a further step is taken by using a remote sensing device for redirecting the wake of a wind turbine. A controller is proposed which uses the yaw actuator of a wind turbine to steer the wake center of the turbine to a desired position. The wake propagation from the wind turbine to the measurement location is modeled with a time delay. This forms a challenging problem for controller design. The controller follows the idea of the internal model principle and uses a model to predict the system behavior avoiding an overestimation of the error. Further, an adaptive filter is proposed in order to filter uncontrollable frequencies from the wake center estimation. The estimation from lidar measurement data is assumed to be perfect. Closed-loop simulations are conducted using the nominal system and a wind farm simulation tool, which was adapted to the scenario. The results are compared to the uncontrolled baseline case and a statically applied yaw offset. They show an increase in the total power output of the wind farm. Together with wake tracking methods, the approach can be considered as a promising step towards closed-loop wind farm control.
\end{abstract}

\section{INTRODUCTION}

In future, wind energy is going to play an important role in global energy production. However, the density of installed wind power will increase in the near future with limited available space in highly windy areas. This leads not only to more wind farms, but also to a dense spacing within the wind farm. An optimization algorithm, which includes the interactions between the wind turbines, can help to find optimal park layouts for a given area, see [1]. New control concepts can help to increase the total power output of existing and new wind farms by adjusting the wake. The wind speed in the wake of a wind turbine is reduced with respect to the free stream and recovers depending on atmospheric conditions. Additionally, the turbulence in the wake is increased. If a wind turbine is hit by a wake from a wind turbine located upwind, the wind turbine produces less power and is faced higher structural loads because of the increased turbulence, see [2].

Quantifying the wake deficit and the increased turbulence in a wake have been of interest for years. Different models have been developed to address the different phenomena the velocity deficit and the increased turbulence intensity.

The authors are with Stuttgart Wind Energy (SWE), University of Stuttgart, Stuttgart, Germany raacheifb. uni-stuttgart.de
They differ in complexity and computational effort and vary from pure empirical ones, to data driven models, to modeling the physics within the wake. Often the models are steady state models, which means they describe the interaction in a static manner and no wake and wind propagation is modeled. This is due to the fact that they are mainly used to predict the power output and optimize it with respect to the layout and the site conditions. In the past years, the need of faster wind farm simulation tools is grown because of addressing the described issues in a wind farm. Simulation of flow phenomena in a wind farm on a higher level of fidelity is based on Computational Fluid Dynamics (CFD), most commonly Large Eddy Simulations (LES) are applied. In recent years, researchers have been modeling the Fluid-StructureInteraction (FSI) by including structural flexibility of the rotor blades and tower, see [3], [4], and [2]. This approach is computationally very expensive, but gives the possibility to simulate the effect of wakes on loads of downstream turbines. In addition, vortex methods based on potential flow theory are applied more and more for wake modeling. This approach has the potential of reducing the computational effort significantly while maintaining sufficient resolution of predominant flow phenomena. Another category in flow interaction models for wind farm simulations are empirical models that are used to describe the different phenomena which occur in a farm. Namely, the ambient wind field and the wake model. In this study, the simulation tool SimWindFarm is used and modified to also describe yaw redirecting, see [5]. More details about the included models are described in Sec. III.

In relation to wind turbine control, the same two goals are valid for wind farm control: 1) maximization of the total power and 2) reduction of the structural loads. These goals were addressed in research with different approaches: 1) axial induction based wind farm control is proposed and investigated and 2) an approach was introduced to redirects the wake.

Axial induction control aims at manipulating the axial induction by the blade pitch or torque actuator and steering the wind turbine to a lower production level. This results in a weaker wake deficit and aims at minimizing structural load effects on the downwind wind turbines. The effects on the overall energy capture of the wind farm is not clear, yet, see [6].

The redirecting approach aims to redirect the wake direction by either individual pitching or by yawing the wind turbine. Therefore, the wake interaction is avoided and the wind turbine downwind can operate in the optimal case in 
free stream condition. Different investigations have shown that the wake can be redirected up to 0.54 times the rotor diameter by yawing it up to $40 \mathrm{deg}$, see [7]. Simulation results of using this approach are promising and show an increase in power, see [8] and [9].

In this work, a controller is proposed that assists the wake redirecting approach by steering the wake center with a feedback controller to a desired position. In [8], the optimal yaw angles are computed using a reduced wake model with simplified atmospheric conditions and applied in an open loop framework. However, model uncertainties can yield to undesired behavior. Therefore, only applying the optimized yaw angles does not lead to a robust implementation. Thus, this contribution can be seen as a step toward a realization of a closed-loop wake redirecting.

The paper is structured as follows: First, the concept of lidar-based wake redirecting is introduced. Then, the simulation model and modifications are described in Section III. Third, in Section IV a reduced model for the controller design is derived and the controller is presented in Section V. Section VI contains the simulation results and finally, a conclusion is given in Section VII.

\section{APPROACH}

In this work, a closed-loop controller is introduced that uses the estimation of the wake center to control the wake center position. It proposes a feedback controller to stabilize the wake center at a desired position and aims to compensate uncertainties in the wake model.

The remote sensing measurement device lidar (LIght Detection and Ranging) has been used in wind energy in the past years, mainly for resource assessment. Lately, it has been successfully used for providing wind speed predictions for wind turbine control, e.g. for feed forward control [10], [11]. Placing a lidar on a wind turbine facing downwind the device can be used for tracking the wake and identifying wake parameters, see [12]. This approach can be used to analyze wake characteristics but also for control purposes. In this work, it is assumed that the wake center can be perfectly estimated. Fig. 1 shows the proposed closed-loop setup. The estimated wake center position from lidar measurement data, $y_{L}$, is fed back into the controller. The controller sets the demanded yaw input, $\gamma_{d e m}$, to control the wake center.

The wake propagation from the wind turbine to the measurement location is modeled with a time delay. The Smith Predictor approach has shown promising results for controlling delayed systems. The basic idea of a Smith Predictor is the concept of internal model control. A model is used to predict the system behavior without delay. The controller gets an immediate feedback through the prediction model. Additionally, the real measured error is fed back. For the design of a wake redirecting controller, the wake model and the yaw actuator model are the important ones, see Fig. 1. They are merged in the system $\Psi$ with the demanded yaw angle, $\gamma_{d e m}$, as input and the measured wake center, $y_{L}$, as output.

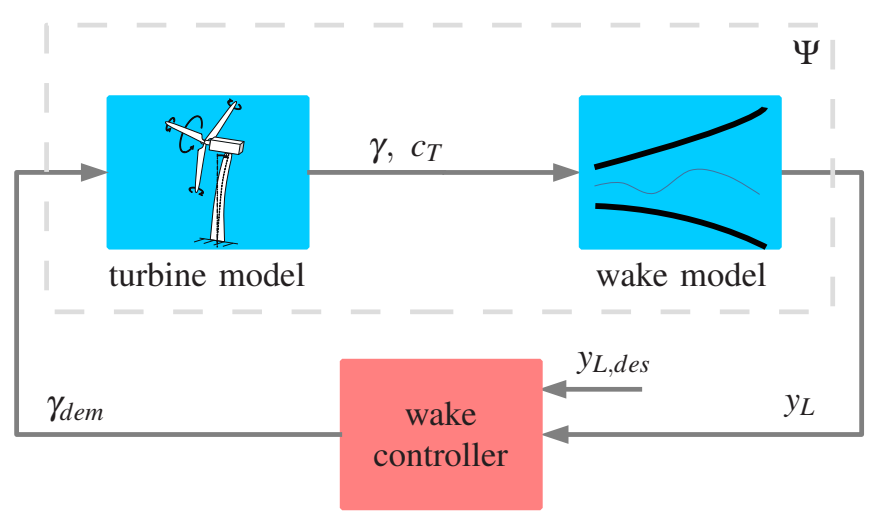

Fig. 1: The closed-loop system. The wake center position, $y_{L}$, is estimated from lidar measurement data and compared to the desired wake center $y_{L, \text { des }}$. The controller sets the yaw input $\gamma_{d e m}$ of the wind turbine to steer the wake center to the demanded position.

\section{Modeling - Simulation Model}

It is important to have a capable simulation model for testing wind farm controllers. It has to fulfill time efficiency issues as well as describing accurately interaction effects of the wind turbines. Here, an engineering model is used that was introduced in [5] and is called SimWindFarm (SWF). In the following, submodules of the simulation model and the necessary adjustments are presented.

\section{A. Turbine Model}

Aero-elastic codes are commonly used to simulate wind turbines for design load calculations. In those simulation codes, the blade element theory is used to calculate the aerodynamic forces effecting the structure. Further, the structure is modeled with different complexity. In SWF, a reduced wind turbine model is used to simulate several wind turbines. A wind turbine is described with multiple degrees of freedom (DOF), e.g. the rotor motion, the fore-aft motion, the drivetrain, the generator, the pitch system and the yaw angle. In the following, the most important dynamics, the rotor motion and the fore-aft motion are briefly described. Further, the new added yaw actuator is presented. For a detailed description of the system and its dynamics see [5].

The rotor motion $\Omega$ can be written, using Euler's law for rotational motion, as

$$
J \dot{\Omega}=M_{a}-M_{g}=\frac{1}{2} \rho \pi R^{2} \frac{c_{P}}{\Omega} v_{0}^{3}-M_{g},
$$

with $M_{a}$ and $M_{g}$ the aerodynamic and generator torque, respectively, $J$ the inertia of all rotational parts, $\rho$ the air density, $R$ the rotor radius, $c_{P}$ the power coefficient, and $v_{0}$ the rotor effective wind speed. The fore-aft motion is modeled using a mass-spring-damper system and the aerodynamic thrust force

$$
F_{a}=\frac{1}{2} \rho \pi R^{2} c_{T} v_{0}^{2}
$$


to

$$
m \ddot{x}_{T}=F_{a}-k_{T} x_{T}-d_{T} \dot{x}_{T}
$$

with the thrust coefficient $c_{T}$, effective tower mass $m, k_{T}$ the spring constant and $d_{T}$ the damping constant. In the aerodynamic coefficients $c_{P}$ and $c_{T}$, the power extraction and the aerodynamic interaction are comprised. They depend on the rotor effective wind speed, the rotor motion and the pitch angle.

The new introduced yaw DOF is defined as an relative offset to the wind direction. Its dynamic is modeled with a second order differential equation

$$
\ddot{\gamma}+2 D \omega \dot{\gamma}+\omega^{2} \gamma=\omega^{2} \gamma_{d e m}
$$

with the undamped eigen frequency $\omega$ and the damping $D$.

\section{B. Turbine Controller}

The implemented wind turbines are classically controlled with a pitch controller and a generator torque controller. There are two different operation regions for the wind turbine controller: In region 2 , the primal goal is to produce maximum power. In region 3, maintaining constant rotor speed is the primal goal while extracting constant electrical power. The $k \Omega^{2}$ control law for the torque controller in region 2 and a gain scheduled PI pitch controller with constant power in region 3 are used. For more details on the controller see, [5] and [13].

\section{Wind Field}

In the simulation code, the ambient wind field is generated using spectral relationships between points in the wind field. Both, the longitudinal and transversal wind components are modeled. The Kaimal spectrum is used for calculating the turbulence. Further, the coherence between two turbines is calculated according to [14]. This yields the cross spectrum

$$
S_{i k}(f)=C_{i k}(f) \sqrt{S_{i i}(f) S_{k k}(f)} \exp \left(-j 2 \pi f \tau_{i k}\right)
$$

with the coherence $C_{i k}$ between turbine $i$ and $k$, the auto spectra $S_{i i}$ and $S_{k k}$ at turbine $i$ and $k$, respectively, and the time delay $\tau_{i k}$ from turbine $i$ to $k$.

\section{Wake Model}

In the next part, the wake expansion, the wake center, and the wake deficit are briefly described. A detailed description on all wake effects and also the wind field generation is given in the manual of the simulation tool and the referred publications, see [5], [15], [16].

1) Wake Expansion: According to [16], the wake expansion diameter at the distance $d$ downwind is given by

$$
D_{\text {wake }}(d)=D\left(\beta\left(c_{T}\right)^{\frac{k}{2}}+\alpha \frac{d}{D}\right)
$$

with the parameter $\alpha=0.5$ and $k=2$ and the function

$$
\beta\left(c_{T}\right)=\frac{1+\sqrt{1-c_{T}}}{2 \sqrt{1-c_{T}}}
$$

where $D$ is the rotor diameter and $c_{T}$ is the aerodynamic thrust coefficient.
2) Wake Center: The wake center is described by a passive tracer with the time shift according to the mean wind speed, $v_{\text {mean }}$. This means, a change in wake properties at time $t_{1}$ is affecting a downwind turbine at distance $d$ at time

$$
t_{2}=t_{1}+\frac{d}{u_{\text {mean }}} \text {. }
$$

Further, the wake center meanders with the mean lateral wind speed at distance $d$. This yields the wake center $y_{\text {meander }}(d)$.

3) Wake Deficit: The velocity deficit caused by a wind turbine is one of the main interactive effects between wind turbines. According to [16], the velocity in the wake at the downwind distance $d$ is given by

$$
U(d) \approx\left(1-\frac{c_{T}}{2} \frac{D^{2}}{D_{\text {wake }}(d)}\right) U_{0}
$$

with the ambient wind speed $U_{0}$ and the thrust coefficient $c_{T}$. In oder to combine the wakes of several wind turbines, they are merged with the following rule:

$$
c_{n+1}=1-\left(\frac{D_{\text {wake }, n}^{2}(d)}{D_{\text {wake }, n+1}^{2}(d)}\left(1-c_{n}\right)+\frac{c_{T, n} D^{2}(d)}{2 D_{\text {wake }, n+1}^{2}(d)} c_{n}\right)
$$

where $c_{n+1}=\frac{U_{n+1}}{U_{0}}$ is the wake deficit at turbine $n+1$. The indexes denote the properties of the different turbines and their wake. For a detailed description, see [16]. To obtain a more realistic approach with respect to the shape of a wake and partial wake situation, the wake center position is further taken into account to scale the wake deficit. Therefore, Gaussian shape wake deficit is assumed and based on the wake center position the wake deficit is moved.

4) Wake Deflection: In order to describe a wake deflection caused by a yaw misalignment $\gamma$, this phenomenon is additionally modeled. The relationship is derived in the study of [17] and was successfully used in an optimization of the yaw angles for a wind farm in [8].

The yaw induced deflection at the downwind position $d$ is according to [8]

$$
\begin{aligned}
\delta_{\text {yaw }}\left(d, c_{T}, \gamma\right) & =-\xi_{\text {init }}\left(c_{T}, \gamma\right) \frac{D}{30 k_{d}}\left[15\left(1-\frac{1}{1+\frac{2 k_{d} d}{D}}\right)\right. \\
& \left.+\xi_{\text {init }}\left(c_{T}, \gamma\right)^{2}\left(1-\frac{1}{\left(1+\frac{2 k_{d} d}{D}\right)^{5}}\right)\right]
\end{aligned}
$$

with the initial angle of the wake at the rotor

$$
\xi_{\text {init }}\left(c_{T}, \gamma\right)=\frac{1}{2} \cos ^{2}(\gamma) \sin (\gamma) c_{T}
$$

and the model parameter $k_{d}$, which defines the sensitivity of the wake deflection to yaw. The wake model (11) shows the following behavior for the limits of the downwind distance, d,

$$
\begin{aligned}
& d=0 \Rightarrow \delta_{\text {yaw }}\left(d, c_{T}, \gamma\right)=0 \\
& d \rightarrow \infty \Rightarrow \\
& \quad \delta_{\text {yaw }}\left(d, c_{T}, \gamma\right) \rightarrow-\xi_{\text {init }}\left(c_{T}, \gamma\right) D \frac{15+\xi_{\text {init }}\left(c_{T}, \gamma\right)^{2}}{30 k_{d}}
\end{aligned}
$$


Further, a rotational induced wake deflection is modeled according to [7]. At the downwind position $d$ the induced deflection is

$$
\delta_{\text {rot }}(d)=a_{d}+b_{d} d,
$$

with the empirical coefficients $a_{d}$ and $b_{d}$. Thus, at the downwind position $d$ the relative wake center position results to

$$
y_{\text {wake }}(d)=y_{\text {meander }}(d)+\delta_{\text {rot }}(d)+\delta_{\text {yaw }}\left(d, c_{T}, \gamma\right) .
$$

\section{E. Lidar Wake Tracking}

In [12], a lidar wake tracking method was introduced. Here, perfect wake center estimation is assumed to focus on the controller and evaluate the ability of the control approach. Therefore, it is assumed that the the estimated wake center position, $y_{L}$, at the downwind distance, $d_{\text {Lidar }}$, is the exact wake center position,

$$
y_{L}=y_{\text {wake }}\left(d_{\text {Lidar }}\right) .
$$

\section{Modeling - InTERnAL MODEL}

The yaw actuator, the wake deflection model and the wake propagation are considered for the internal controller design model. In the following, they are described and the simplifications are highlighted:

The yaw actuator described in (4) is used in the internal model without any modification.

In the wake deflection model (12) a simplification is made. Here, a constant $c_{T}=c_{T, \text { const }}$ is assumed. This is reasonable since we are focusing on below rated wind speed situations where $c_{T}$ is almost constant. Further, only (11) is used and the rotational offset (15) is neglected.

Assuming perfect wake center estimation, the wake center, $y_{L}$, is estimated at the downwind distance, $d_{\text {Lidar }}$. Due to the wake propagation, the time delay $\tau$ is introduced and the reaction of the wake center is measured with that delay.

Altogether, this yields a controller design model $\widetilde{\Psi}$ of the system $\Psi$ :

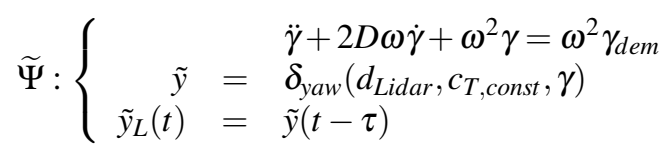

To summarize, the internal model consists of the yaw actuator model, the approximation of the wake deflection and a time delay. A constant thrust coefficient $c_{T}$ is assumed, the wake deflection due to the rotation is neglected, and there is no model of the wake meandering.

\section{CONTROLLER DESIGN}

The primal goal of the wake controller is to steer the wake center to a desired point by deflecting the wake in a way such that the wake center is at the desired position. In Section IV a reduced model for the controller has been derived. In the following, the proposed control strategy is adapted to the given problem. Figure 2 describes the proposed controller concept and its different parts.

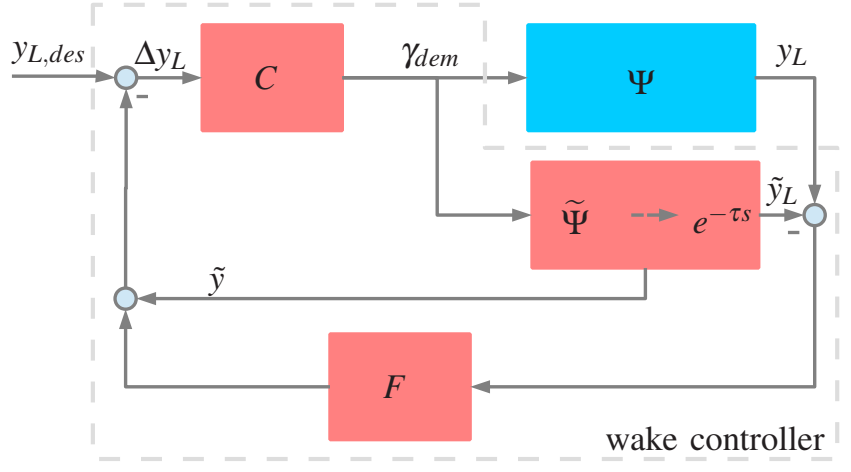

Fig. 2: The wake controller and its paths: the controller $C$, the internal model $\widetilde{\psi}$, and the filter $F$.

The controller $C$ uses the demanded yaw input to control the measured wake center $y_{L}$. The internal model $\widetilde{\psi}$ is used to predict the deflection of the wake center. The measured wake center $y_{L}$ is compared to the predicted $\tilde{y}_{L}$ and filtered with the filter $F$ to remove frequencies that are not controllable. The filtered signal is fed back to the controller as well as the immediate predicted wake center $\tilde{y}$.

\section{A. Controller}

The controller $C$ consists of a proportional-integral (PI) controller and is designed such that it meets the desired closed-loop performance with the dynamic of (18). A phase margin of $60 \mathrm{deg}$ and a closed-loop bandwidth of $\omega_{C L}=\frac{1}{\tau}$ are set. This yields a controller of the form

$$
u=K_{p}\left(\Delta y_{L}+\frac{1}{T_{i}} \int \Delta y_{L} d t\right)
$$

with the proportional gain $K_{p}$ and the time constant $T_{i}$.

Remark. It is important to remark that the designed controller is only able to act on disturbances at frequencies lower than the dead time, the delay of the system. This means that wake meandering effects at high frequencies can not be suppressed with this approach, the static position, however, is controlled. For this reason, an adaptive low pass filter is designed based on the delay $\tau$ which changes with respect to the wind speed.

\section{B. Adaptive Filter}

The filter is set up depending on the time delay $\tau$, which depends on the mean wind speed $u_{\text {mean }}$. It damps all frequencies which can not be controlled. Therefore, the cutoff frequency is set to $\omega_{\text {filter }}=\frac{\pi}{8 \tau}$ and a second order butterworth low-pass filter is designed.

\section{RESULTS}

\section{A. Results using the Controller Design Model}

In the following, the closed-loop behavior is analyzed and two step response simulation results are presented. A mean wind speed of $8 \mathrm{~m} / \mathrm{s}$ and a lidar measurement distance of $1 \mathrm{D}$, with $D=126 \mathrm{~m}$, is assumed. Thus, the dead time results in $\tau=15.75 \mathrm{~s}$. The controller and the filter are set up as previously described. For the first simulation, no 

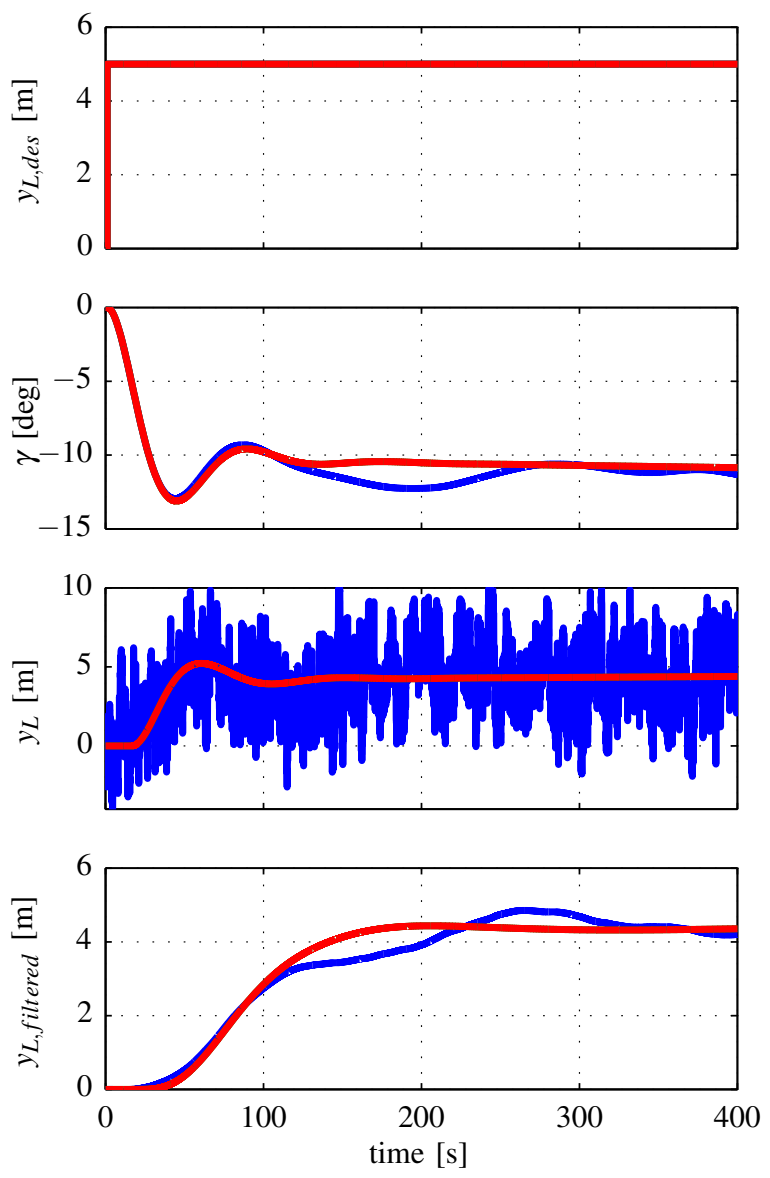

Fig. 3: A comparison of two step response simulations with the nominal closed-loop system. There is no wake meandering in the first simulation, (red). The nominal system is disturbed by an additional meandering in the second simulation, (blue).

wake meandering is assumed. Thus, there is no disturbance acting on the output $y_{L}$. For the second simulation a wake meandering between $\pm 5 \mathrm{~m}$ is used to disturb the wake center. The results in Fig. 3 show, that the controller setup is able to steer the wake center to the desired position, although the wake center is meandering.

\section{B. Results using the Simulation Model}

To evaluate the ability of increasing the power output, a $2 \times 3$ wind farm layout is simulated with 6 NREL $5 \mathrm{MW}$ turbines. The downwind spacing is $5 \mathrm{D}$ and the lateral spacing $3 \mathrm{D}$. A turbulent wind field of $1000 \mathrm{~s}$ with a mean wind speed $u_{\text {mean }}=8 \mathrm{~m} / \mathrm{s}$ and a rotor effective turbulence intensity of $1 \%$ is used. The desired wake positions are approximated using the results of [8].

First, in Fig. 4 the functionality of the controller in the simulation environment is verified by analyzing the wake center $y_{L}$, the filtered wake center $y_{L \text {,filtered }}$, the yaw angle $\gamma$ and the power output of the first downwind turbine (turbine 2 ). The wake center is steered to the desired position. In the power output of turbine 2 at time $t \approx 180 \mathrm{~s}$, the wake turbine 1: uncontrolled (blue), closed-loop (red)
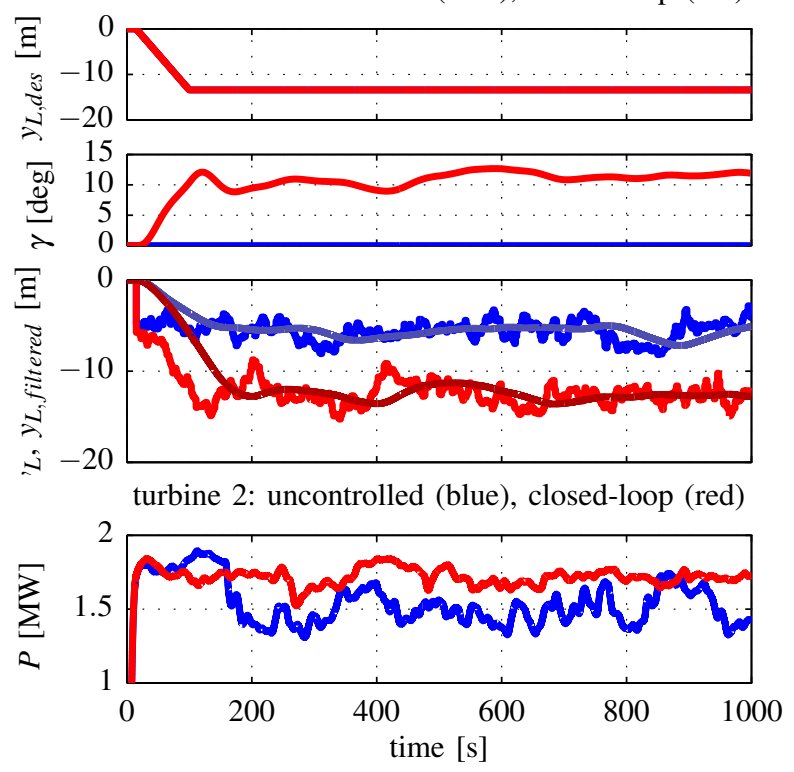

Fig. 4: A comparison between a simulation where the redirecting controller is off, (blue), and a simulation where the wake is redirected, (red). The wake center is redirected to the desired value, (gray). In the last plot, the power output of turbine 2 is shown. The decrease in power is observed in the case, when the wake hit the second turbine.

of the first turbine is evolved over the total wind domain and the calculations are started. Thus, we see that for the uncontrolled simulation (blue) the power drops. For the controlled simulation the power is nearly kept constant.

Second, three simulations are carried out to evaluate the closed-loop approach: 1) a simulation without a wind farm control strategy, 2) a simulation, where the static yaw angles are applied in open-loop, and 3) a simulation, where the wakes are controlled in the proposed closed-loop. The total power of the wind farm for the three simulation cases is presented in Fig. 5. The overall power output of the closedloop controlled wind increased by approximately $4.5 \%$. Compared to the open-loop approach, the total power output is almost the same. However, it has the benefits of a closedloop controller such as compensate uncertainties in the wake model.

\section{CONCLUSION}

In this contribution a feedback controller is presented to control the wake center of a wind turbine assuming perfect estimation of the wake center. First, the simulation model is described. Then, a reduced controller design model is derived which is used in the controller to predict the wake behavior. This is necessary because the wake propagation to the measurement position of the lidar introduces a time delay. An adaptive filter is designed to remove the uncontrollable frequencies in the wake center dynamics. This is necessary because the wake meandering is disturbing the wake center position and the time delay makes it infeasible to control 


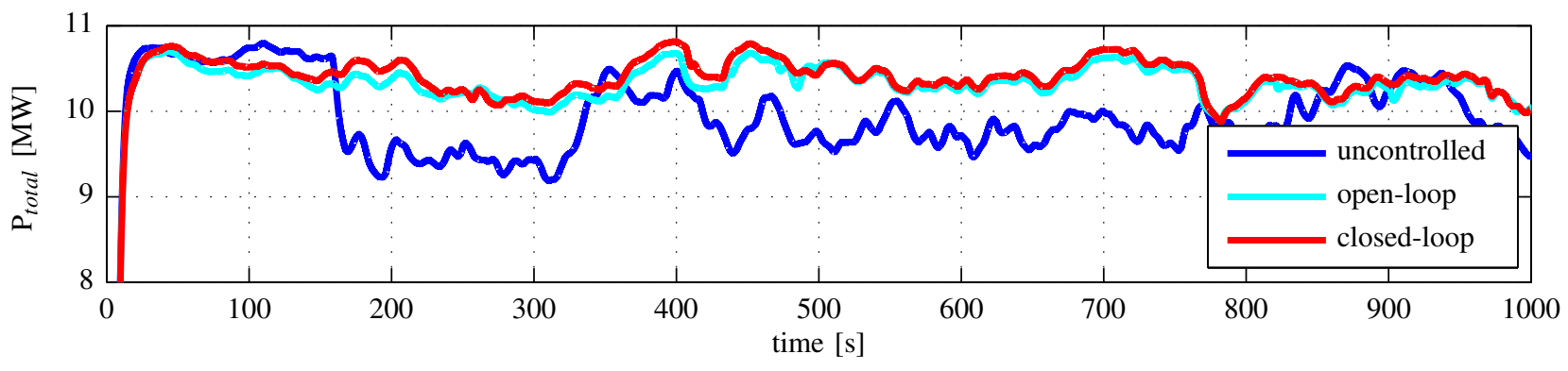

Fig. 5: Comparison of the total power output of the wind farm of three controller scenarios. Light blue depicts the greedy control strategy where all wind turbines are perfectly aligned with the wind direction. In red, the presented controller is applied. The total power does not drop when the wakes affect the downwind turbines. In blue, the total power of a simulation is shown in which precomputed static yaw angles are applied. The power output is almost the same than the controlled case.

the meandering. The concept is tested in the wind farm simulation tool SimWindFarm and test simulations with 6 wind turbines in a $2 \times 3$ wind farm layout are performed. Altogether, the approach shows promising results in the simulation by increasing the total power of the wind farm. Together with wake tracking methods, the approach can be considered as a step towards closed-loop wind farm control. In the future, the controller shall be tested in simulation models with higher fidelity. Further, a field testing of wake redirecting is pursued.

\section{ACKNOWLEDGMENT}

The main author specially want to thank Pieter Gebraad for the fruitful discussions and his help to get a deeper understanding of the topic. Further, the valuable remarks of Torben Knudsen are gratefully appreciated.

\section{REFERENCES}

[1] P. A. Fleming, A. Ning, P. M. O. Gebraad, and K. Dykes, "Wind plant system engineering through optimization of layout and yaw control," Wind Energy, pp. 1-16, 2016.

[2] B. Luhmann, F. Borisade, S. Raach, and P. Cheng, "Coupled AeroElastic Multi-Body Simulation of Two-Bladed Wind Turbines in Wind Park Arrays," in Proceedings of the AIAA conference, 2016.

[3] M. Churchfield, S. Lee, and P. Moriarty, "Overview of the Simulator for Offshore Wind Farm Application SOWFA," 2012.

[4] F. Beyer, B. Luhmann, S. Raach, and P. W. Cheng, "Shadow Effects in an Offshore Wind Farm - Potential of Vortex Methods for Wake Modelling," in Proceedings of the DEWEK, 2015.

[5] J. D. Grunnet, M. Soltani, T. Knudsen, M. Kragelund, and T. Bak, "Aeolus Toolbox for Dynamic Wind Farm Model, Simulation and Control," in Proceedings of the European Wind Energy Conference, 2010.

[6] J. Annoni, P. M. Gebraad, A. K. Scholbrock, P. A. Fleming, and J.-W. v. Wingerden, "Analysis of axial-induction-based wind plant control using an engineering and a high-order wind plant model," Wind Energy, 2015.
[7] P. A. Fleming, P. M. Gebraad, S. Lee, J.-W. van Wingerden, K. Johnson, M. Churchfield, J. Michalakes, P. Spalart, and P. Moriarty, "Evaluating techniques for redirecting turbine wakes using SOWFA," Renewable Energy, vol. 70, pp. 211-218, 2014.

[8] P. M. O. Gebraad, F. W. Teeuwisse, J. van Wingerden, P. Fleming, S. D. Ruben, J. R. Marden, and L. Y. Pao, "Wind plant power optimization through yaw control using a parametric model for wake effectsa CFD simulation study," Wind Energy, 2014.

[9] P. Fleming, P. M. Gebraad, S. Lee, J.-W. Wingerden, K. Johnson, M. Churchfield, J. Michalakes, P. Spalart, and P. Moriarty, "Simulation comparison of wake mitigation control strategies for a two-turbine case," Wind Energy, 2014.

[10] D. Schlipf, D. J. Schlipf, and M. Kühn, "Nonlinear model predictive control of wind turbines using LIDAR," Wind Energy, vol. 16, no. 7, pp. 1107-1129, 2013.

[11] A. Scholbrock, P. Fleming, L. Fingersh, A. Wright, D. Schlipf, F. Haizmann, and F. Belen, "Field testing LIDAR based feed-forward controls on the NREL controls advanced research turbine," in 51th AIAA Aerospace Sciences Meeting Including the New Horizons Forum and Aerospace Exposition, 2013.

[12] S. Raach, D. Schlipf, J. J. Trujillo, and P. W. Cheng, "Model-based wake tracking using lidar measurements for wind farm control," in Windfarms 2015, Leuven, Belgium, 2015.

[13] J. Jonkman, S. Butterfield, W. Musial, and G. Scott, "Definition of a 5-MW Reference Wind Turbine for Offshore System Development," National Renewable Energy Laboratory, Tech. Rep., 2009.

[14] P. Sørensen, A. D. Hansen, P. Andr, and C. Rosas, "Wind Models for Simulation of Power Fluctuations from Wind Farms," Journal of Wind Engineering and Industrial Aerodynamics, vol. 90, pp. 13811402, 2002.

[15] G. C. Larsen, H. A. Madsen, K. Thomsen, and T. J. Larsen, "Wake Meandering: A Pragmatic Approach," Wind Energy, vol. 11, pp. 377395, 2008.

[16] S. Frandsen, R. Barthelmie, S. Pryor, O. Rathmann, and S. Larsen, "Analytical Modelling of Wind Speed Deficit in Large Offshore Wind Farms," Wind Energy, vol. 9, no. January, pp. 39-53, 2006.

[17] Á. Jiménez, A. Crespo, and E. Migoya, "Application of a LES technique to characterize the wake deflection of a wind turbine in yaw," Wind energy, vol. 13, no. 6, pp. 559-572, 2010. 